\title{
AN OPTIMAL CONDITION FOR THE LIL FOR TRIGONOMETRIC SERIES
}

\section{BERKES}

ABSTRACt. By a classical theorem (Salem-Zygmund [6], Erdős-Gál [3]), if $\left(n_{k}\right)$ is a sequence of positive integers satisfying $n_{k+1} / n_{k} \geq q>1 \quad(k=1,2, \ldots)$ then $\left(\cos n_{k} x\right)$ obeys the law of the iterated logarithm, i.e.,

$$
\limsup _{N \rightarrow \infty}(N \log \log N)^{-1 / 2} \sum_{k \leq N} \cos n_{k} x=1 \quad \text { a.e. }
$$

It is also known (Takahashi [7, 8]) that the Hadamard gap condition $n_{k+1} / n_{k} \geq$ $q>1$ can be essentially weakened here but the problem of finding the precise gap condition for the LIL (1) has remained open. In this paper we find, using combinatorial methods, an optimal gap condition for the upper half of the LIL, i.e., the inequality $\leq 1$ in (1).

\section{INTRODUCTION}

It is a well-known fact that lacunary subsequences of the trigonometric system behave like sequences of independent random variables. For example, if $\left(n_{k}\right)$ is a sequence of positive integers satisfying the Hadamard gap condition

$$
n_{k+1} / n_{k} \geq q>1 \quad(k=1,2, \ldots)
$$

then by classical results of Salem-Zygmund [5, 6] and Erdős-Gál [3] we have

$$
\begin{gathered}
\lim _{N \rightarrow \infty} \frac{1}{2 \pi} \lambda\left\{0 \leq x \leq 2 \pi: \sum_{k \leq N} \cos n_{k} x<t \sqrt{N / 2}\right\} \\
=(2 \pi)^{-1 / 2} \int_{-\infty}^{t} e^{-u^{2} / 2} d u
\end{gathered}
$$

and

$$
\limsup _{N \rightarrow \infty}(N \log \log N)^{-1 / 2} \sum_{k \leq N} \cos n_{k} x=1 \quad \text { a.e. }
$$

Actually, the exponential growth condition (1.1) can be weakened in the above results as the following theorem of Erdős [2] shows:

Received by the editors November 1, 1993.

1991 Mathematics Subject Classification. Primary 42A55, 60F15.

Research supported by Hungarian National Foundation for Scientific Research, Grant No. 1905. 
Theorem A. Let $\left(n_{k}\right)$ be a sequence of positive integers satisfying

$$
n_{k+1} / n_{k} \geq 1+c_{k} / \sqrt{k}, \quad c_{k} \rightarrow \infty .
$$

Then the sequence $\left(\cos n_{k} x\right)$ satisfies the central limit theorem (1.2). On the other hand, for each $c>0$ there exists a sequence $\left(n_{k}\right)$ of positive integers such that

$$
n_{k+1} / n_{k} \geq 1+c / \sqrt{k} \quad\left(k \geq k_{0}\right)
$$

but the central limit theorem (1.2) is not valid.

In terms of the growth speed of $\left(n_{k}\right)$, Theorem A gives a precise criterion for $\left(\cos n_{k} x\right)$ to satisfy the central limit theorem. No similarly complete result exists in the case of the law of the iterated logarithm and in fact for subexponentially growing $\left(n_{k}\right)$ the LIL turns out to be a much more delicate problem than the CLT. Takahashi proved (see $[7,8])$ that the LIL (1.3) holds if $\left(n_{k}\right)$ satisfies (1.4) with $c_{k}>k^{\gamma}$ for some $\gamma>0$; in [1] we showed that this rate can be weakened to $c_{k}>(\log \log k)^{\gamma}, \gamma>\gamma_{0}$, and this result is essentially optimal in the sense that there exist sequences $\left(n_{k}\right)$ satisfying (1.4) with $c_{k}=(\log \log k)^{1 / 2}$ such that the LIL (1.3) is false. The results of [1] show that for $c_{k}=(\log \log k)^{\gamma}$ the a.s. behavior of $\left(\cos n_{k} x\right)$ is very delicate: small changes of $\gamma$ can lead to radical changes in the fluctuational properties of $\left(\cos n_{k} x\right)$, moreover, in the domain $c_{k}=(\log \log k)^{\gamma}$ the sequence $\left(\cos n_{k} x\right)$ exhibits highly unusual forms of "fractional" LIL behavior in the sense that it satisfies some forms of the LIL but fails similar, closely related LIL type results. The methods of [1] are not strong enough to determine the precise constants $\gamma$ required for various forms of the LIL (such as the Kolmogorov-Erdös-Feller-Petrovski test, Chung type LIL's etc.); in fact even the optimal value of $\gamma$ required for the ordinary LIL (1.3) has remained undetermined. The purpose of this paper is to improve the combinatorial tools of [1] and to find the precise gap condition for the upper half of the LIL. More precisely, we shall prove the following result.

Theorem. Let $\left(n_{k}\right)$ be a sequence of positive integers satisfying

$$
n_{k+1} / n_{k} \geq 1+(\log \log k)^{\alpha} / \sqrt{k} \quad\left(k \geq k_{0}\right)
$$

for some $\alpha>1 / 2$. Then $\left(\cos n_{k} x\right)$ satisfies

$$
\limsup _{N \rightarrow \infty}(N \log \log N)^{-1 / 2}\left|\sum_{k \leq N} \cos n_{k} x\right| \leq 1 \quad \text { a.e. }
$$

On the other hand, there exists a sequence $\left(n_{k}\right)$ of positive integers satisfying (1.5) with $\alpha=1 / 2$ such that (1.6) is not valid.

The second half of this theorem was proved in [1]; in fact, the example constructed in the proof of Theorem 2 of [1] shows that (1.5) with $\alpha=1 / 2$ permits rather pathological LIL behavior of $\left(\cos n_{k} x\right)$ : it can happen, e.g., that $(N \log \log N)^{-1 / 2} \sum_{k \leq N} \cos n_{k} x$ has a nonsymmetric cluster set, with its $\lim \sup <1$ and $\lim \inf <-1$ a.e.

It seems likely that (1.5) with $\alpha>1 / 2$ actually implies (1.6) with lim sup $=1$ but this remains open. On the other hand, the results of [1] show that (1.5) with $\alpha>5 / 2$ implies (1.6) with $\lim \sup =1$. 
It should be noted that the validity of the LIL (1.6) (or even of (1.3)) does not imply that the partial sum behavior of $\left(\cos n_{k} x\right)$ is exactly the same as that of independent r.v.'s. In [1] we constructed a sequence $\left(n_{k}\right)$ of integers such that (1.2), (1.3) (and in fact (1.5) with $\alpha>1 / 2$ ) hold and

$$
\sum_{k \leq N} \cos n_{k} x<(N \log \log N)^{1 / 2} \quad \text { a.e. for } N \geq N_{0}(x) .
$$

On the other hand, if $X_{k}$ are independent r.v.'s with $X_{k}$ distributed as $\cos n_{k} x$ (and hence the $X_{k}$ are i.i.d) then by the classical Kolmogorov-Erdős-FellerPetrovski test (see, e.g., [4]) we have for any increasing function $\varphi:(0, \infty) \rightarrow$ $(0, \infty)$

$$
P\left\{\sum_{k \leq N} X_{k}<\sqrt{N / 2} \varphi(N) \text { a.s. for } N \geq N_{0}\right\}=0 \text { or } 1
$$

according as

$$
\sum_{N \geq 1} \frac{\varphi(N)}{N} \exp \left\{-\frac{1}{2} \varphi^{2}(N)\right\}<+\infty \text { or }=+\infty
$$

In particular,

$$
\sum_{k \leq N} X_{k} \geq(N \log \log N)^{1 / 2} \quad \text { a.s. for infinitely many } N
$$

and thus the partial sum behavior of $\left(\cos n_{k} x\right)$ is different from that of $\left(X_{k}\right)$. By the main result of [1], $\left(\cos n_{k} x\right)$ satisfies the Kolmogorov-Erdös-FellerPetrovski test if (1.5) holds with a sufficiently large $\alpha$ and thus the above pathological phenomenon cannot occur for large $\alpha$. It is still open what is the smallest value of $\alpha$ implying the Kolmogorov-Erdős-Feller-Petrovski test for $\left(\cos n_{k} x\right)$; in [1] we showed that $\alpha>5 / 2$ suffices while $\alpha \leq 3 / 2$ does not. A comparison of the results of [1] and [4] suggests strongly that the critical value is $\alpha_{0}=3 / 2$ i.e., the Kolmogorov-Erdős-Feller-Petrovski test holds for $\alpha>3 / 2$. (See in this respect also the remark at the end of our paper.)

The proof of our theorem will be combinatorial; in fact we shall deduce our result from estimates for the number of solutions of the diophantine equation

$$
\pm n_{i_{1}} \pm n_{i_{2}} \pm \cdots \pm n_{i_{p}}=0, \quad(1-\delta) N \leq i_{1}, \ldots, i_{p} \leq N .
$$

This technique goes back (at least) to Erdős-Gál [3], but the subexponential domain presents considerable difficulties and we shall need essential improvement of earlier results to get the LIL (1.6) under the sharp gap condition (1.5), $\alpha>1 / 2$.

\section{A COMBINATORIAL LEMMA}

The purpose of this section is to prove the following combinatorial statement which is the key step in the proof of our theorem.

Main Lemma. Let $\left\{n_{j}, 1 \leq j \leq N\right\}$ be a finite sequence of positive integers satisfying

$$
n_{j+1} / n_{j} \geq 1+c / \sqrt{j}, \quad 1 \leq j \leq N-1 .
$$


Further let $p \geq 2$ be an even integer and assume that

$$
N \geq c^{2}, \quad p \leq 4 \log \log N, \quad c \geq p^{1 / 2+\varepsilon}
$$

for some $0<\varepsilon \leq 1$. Then for any integer $d$ and $0<\delta \leq 1$ the number of solutions of the equation

$$
\pm n_{i_{1}} \pm n_{i_{2}} \pm \cdots \pm n_{i_{p}}=d, \quad(1-\delta) N \leq i_{1}, \ldots, i_{p} \leq N,
$$

is at most

$$
\exp \left\{\frac{K p^{1-\varepsilon} \log ^{3} p}{\sqrt{\delta}}\right\} \frac{p !}{(p / 2) !}(\delta N)^{p / 2}
$$

provided that $p \geq K$ and $N \geq N_{0}(\delta)$ where $K$ is an absolute constant.

By the number of solutions of (2.3) we mean the number of $2 p$-tuples $\left(i_{1}, \ldots, i_{p}, \varepsilon_{1}, \ldots, \varepsilon_{p}\right)$ where $i_{1}, \ldots, i_{p}$ are integers with $(1-\delta) N \leq i_{1}, \ldots$, $i_{p} \leq N$, the $\varepsilon_{j}$ 's are \pm 1 , and $\varepsilon_{1} n_{i_{1}}+\cdots+\varepsilon_{p} n_{i_{p}}=d$. Call a solution $\left(n_{i_{1}}, \ldots, n_{i_{p}}\right)$ of (2.3) trivial if $d=0$ and among the terms in (2.3) each $n_{j}$ occurs the same number of times with a positive as with a negative sign. It is easy to see that the number of trivial solutions of $(2.3)$ is

$$
(1+o(1)) \frac{p !}{(p / 2) !}(\delta N)^{p / 2}
$$

as $N \rightarrow \infty$, uniformly in $2 \leq p \leq 4 \log \log N$. Hence our main lemma states that for any $d$ the number of solutions of (2.3) exceeds the number of trivial solutions only by a subexponential factor $\exp \left(C p^{\gamma}\right), \gamma<1$. As the example in $\S 4$ will show, for $\varepsilon$ small enough, this subexponential factor cannot be removed (or improved beyond $O\left(p^{2-2 \varepsilon}\right)$ ) even for $d=0$. Hence for $\varepsilon$ small and $p$ large, equation (2.3) with $d=0$ can have many more nontrivial solutions than trivial ones. On the other hand, the proof of the main lemma will show that for $\varepsilon>1$ the subexponential factor in (2.4) can be replaced by $1+O\left(p^{-(\varepsilon-1) / 6}\right)$ or $O\left(p^{-(\varepsilon-1) / 6}\right)$ according as we include or exclude the trivial solutions. In other words, for $\varepsilon>1$ and $p$ large, most solutions of (2.3) with $d=0$ are trivial. The remarkable consequences of this discrepancy will be discussed in $\S 4$.

We turn now to the proof of the main lemma. To simplify the formulas, we assume $\delta=1$; the general case will require only trivial modifications. We break the argument into several steps.

Lemma 1. Let $\left\{n_{j}, 1 \leq j \leq N\right\}$ be a sequence of positive integers satisfying (2.1) and assume $N \geq c^{2}$. Then for any $0<a<b$ the interval $[a, b]$ contains at most

$$
2 c^{-1} \sqrt{N} \log (b / a)+1
$$

terms of the sequence $\left\{n_{j}, 1 \leq j \leq N\right\}$.

Proof. Let $n_{q}$ and $n_{r}$ be the smallest and largest among the $n_{j}$ 's $(1 \leq j \leq N)$ in the interval $[a, b]$. Then $n_{r} / n_{q} \leq b / a$; on the other hand, by $(2.1)$ we have

$$
\frac{n_{r}}{n_{q}} \geq \prod_{j=q}^{r-1}\left(1+\frac{c}{\sqrt{j}}\right) \geq\left(1+\frac{c}{\sqrt{N}}\right)^{r-q} \geq \exp \left\{\frac{c}{2 \sqrt{N}}(r-q)\right\}
$$


using the fact that $1+x \geq e^{x / 2}$ for $0 \leq x \leq 1$. The two estimates for $n_{r} / n_{q}$ imply

$$
\exp \left\{\frac{c}{2 \sqrt{N}}(r-q)\right\} \leq b / a
$$

whence $r-q+1 \leq 2 c^{-1} \sqrt{N} \log (b / a)+1$, as stated.

In what follows, fix an arbitrary sequence $\varepsilon_{1}, \ldots, \varepsilon_{p}$ of \pm 1 's and consider the equation

$$
\varepsilon_{1} n_{i_{1}}+\cdots+\varepsilon_{p} n_{i_{p}}=d, \quad N \geq i_{1} \geq i_{2} \geq \cdots \geq i_{p} \geq 1 .
$$

In other words, in (2.3) we fix the signs and the order of the $i_{k}$ 's. To simplify the writing in the sequel, we introduce some terminology. Given a solution $\left(n_{i_{1}}, \ldots, n_{i_{p}}\right)$ of $(2.5)$, the ratios $n_{i_{k}} / n_{i_{k+1}}$ will be called the gaps in this solution. For any $1 \leq k \leq p-1$, define the positive integer $j_{k}$ by $n_{i_{k}} / n_{i_{k+1}} \asymp 2^{j_{k}}$ where the symbol $a \asymp 2^{j}$ means $2^{j} \leq a<2^{j+1}$. The gap $n_{i_{k}} / n_{i_{k+1}}$ is then called

$$
\begin{array}{ll}
\text { small } & \text { if } p 2^{-j_{k}} \geq 1 / 8, \\
\text { medium } & \text { if } c /(32 \sqrt{N}) \leq p 2^{-j_{k}}<1 / 8, \\
\text { large } & \text { if } p 2^{-j_{k}}<c /(32 / \sqrt{N}) .
\end{array}
$$

In a solution $\left(n_{i_{1}}, \ldots, n_{i_{p}}\right)$ of $(2.5)$, the segment $\left(n_{i_{k}}, \ldots, n_{i_{l}}\right)$ will be called a block if it contains no large gaps but it is preceded and followed by a large gap. (For $k=1$ or $l=p$ there is only one side condition.) A block $\left(n_{i_{k}}, n_{i_{k+1}}\right)$ of length 2 is called trivial if $i_{k}=i_{k+1}$ and $\varepsilon_{k}=-\varepsilon_{k+1}$; otherwise it is called nontrivial.

Lemma 2. Let $2 \leq k \leq p-1$ and consider those solutions of (2.5) where $n_{i_{\nu}} / n_{i_{\nu+1}} \asymp 2^{j_{\nu}} \quad(2 \leq \nu \leq k)$; here $j_{2}, \ldots, j_{k}$ are fixed nonnegative integers. Then, given $n_{i_{1}}, \ldots, n_{i_{k-1}}$, the number of choices for $n_{i_{k}}$ is at most

$$
\begin{cases}3 \sqrt{N} / c & \text { if } p 2^{-j_{k}} \geq 1 / 8 \\ (48 \sqrt{N} / c) \cdot p 2^{-j_{k}} & \text { if } c /(32 \sqrt{N}) \leq p 2^{-j_{k}}<1 / 8 .\end{cases}
$$

Note that the estimates in the first and second line of (2.7) are stated under the conditions that the gap $n_{i_{k}} / n_{i_{k+1}}$ is small or medium.

Proof. By $n_{i_{k-1}} / n_{i_{k}} \asymp 2^{j_{k-1}}$ we have $n_{i_{k}} \in\left[2^{-j_{k-1}-1} n_{i_{k-1}}, 2^{-j_{k-1}} n_{i_{k-1}}\right]$. Hence using Lemma 1 it follows that given $n_{i_{1}}, \ldots, n_{i_{k-1}}$, for $n_{i_{k}}$ we have at most $(2 \sqrt{N} / c) \log 2+1 \leq 3 \sqrt{N} / c$ choices (provided $N \geq c^{2}$ ) no matter which assumption on $p 2^{-j_{k}}$ in (2.7) holds. Assume now that $p 2^{-j_{k}}$ satisfies the assumption in the second line of (2.7). Let $n_{i_{1}}, \ldots, n_{i_{k-1}}$ be given and let $\varepsilon_{1} n_{i_{1}}+\cdots+\varepsilon_{k-1} n_{i_{k-1}}=A$. By $n_{i_{k}} / n_{i_{k+1}} \asymp 2^{j_{k}}$ it follows that the numbers $n_{i_{k+1}}, \ldots, n_{i_{p}}$ are all $\leq n_{i_{k}} \cdot 2^{-j_{k}}$ and thus

$$
\left|\varepsilon_{k+1} n_{i_{k+1}}+\cdots+\varepsilon_{p} n_{i_{p}}\right| \leq p n_{i_{k}} 2^{-j_{k}} .
$$

Hence (2.5) yields

$$
A+\varepsilon_{k} n_{i_{k}}\left(1+\theta p 2^{-j_{k}}\right)=d, \quad|\theta| \leq 1 .
$$


Thus, setting $B=(d-A) / \varepsilon_{k}$ and using $p 2^{-j_{k}}<1 / 8$ and the fact that for $|x| \leq 1 / 2$ we have $(1+x)^{-1}=1+\lambda x$ with $|\lambda| \leq 2$, we get

$$
n_{i_{k}}=B\left(1+\theta p 2^{-j_{k}}\right)^{-1}=B\left(1+\theta^{\prime} p 2^{-j_{k}}\right), \quad\left|\theta^{\prime}\right| \leq 2 .
$$

Here $B \neq 0$ since $n_{i_{k}} \neq 0$. Thus using Lemma 1 and the assumption in the second line of (2.7) it follows that there are at most

$$
\begin{gathered}
(2 \sqrt{N} / c) \cdot \log \frac{1+2 p 2^{-j_{k}}}{1-2 p 2^{-j_{k}}}+1 \leq(2 \sqrt{N} / c) \cdot \log \left(1+8 p 2^{-j_{k}}\right)+1 \\
\leq(16 \sqrt{N} / c) \cdot p 2^{-j_{k}}+1 \leq(48 \sqrt{N} / c) \cdot p 2^{-j_{k}}
\end{gathered}
$$

choices for $n_{i_{k}}$.

Lemma 3. Let $1 \leq k \leq p-1$ and consider those solutions of (2.5) where the gap $n_{i_{k}} / n_{i_{k+1}}$ is large. Then given $n_{i_{1}}, \ldots, n_{i_{k-1}}$ (assuming nothing if $k=1$ ) there is at most one possibility for $n_{i_{k}}$.

This follows similarly as the second estimate in (2.7) in the previous lemma. Since the gap $n_{i_{k}} / n_{i_{k+1}}$ is large, we have $n_{i_{k}} / n_{i_{k+1}} \geq 32 p \sqrt{N} / c$ and thus the numbers $n_{i_{k+1}}, \ldots, n_{i_{p}}$ are all less than $n_{i_{k}} \cdot c /(32 p \sqrt{N})$. Hence with $n_{i_{1}}, \ldots$, $n_{i_{k-1}}$ given and setting $B=(d-A) / \varepsilon_{k}$, it follows as above that

$$
n_{i_{k}} \in[B(1-2 c /(32 \sqrt{N})), B(1+2 c /(32 \sqrt{N}))]
$$

and by Lemma 1 the above interval contains at most

$$
\frac{2 \sqrt{N}}{c} \log \left(1+\frac{8 c}{32 \sqrt{N}}\right)+1 \leq 3 / 2
$$

integers.

Lemma 4. Fix $k \geq 0, s \geq 1$ and consider those solutions of (2.5) where $\left(n_{i_{k+1}}, \ldots, n_{i_{k+s}}\right)$ is a block. Then given $\left(n_{i_{1}}, \ldots, n_{i_{k}}\right)$ (assuming nothing if $k=0)$ the number of choices for the s-tuple $\left(n_{i_{k+1}}, \ldots, n_{i_{k+s}}\right)$ is at most

$$
\frac{(600 \log p)^{s}}{c^{s-2}} N^{s / 2} \quad \text { if } s \geq 3
$$

$$
\begin{array}{ll}
1 & \text { if } s=1, \\
3 \sqrt{N} \log N & \text { if } s=2 \text { and the block }\left(n_{i_{k+1}}, n_{i_{k+2}}\right) \text { is nontrivial, } \\
N & \text { if } s=2 \text { and the block }\left(n_{i_{k+1}}, n_{i_{k+2}}\right) \text { is trivial. }
\end{array}
$$

Proof. With $n_{i_{1}}, \ldots, n_{i_{k}}$ given, set $A=\varepsilon_{1} n_{i_{1}}+\cdots+\varepsilon_{k} n_{i_{k}}$. Then the remaining terms $n_{i_{k+1}}, \ldots, n_{i_{p}}$ satisfy the equation

$$
\varepsilon_{k+1} n_{i_{k+1}}+\cdots+\varepsilon_{p} n_{i_{p}}=d-A
$$

analogous to (2.5). Hence without loss of generality we may assume $k=0$. Also, the estimate in the last line of (2.8) is trivial (since there are at most $N$ choices for $n_{i_{k+1}}=n_{i_{k+2}}$ ) and the estimate in the second line follows from Lemma 3 if $k+1<p$ and is trivial if $k+1=p$. Hence it suffices to prove the estimates in the first and third lines.

In the case $s=2$ the nontriviality of the block $\left(n_{i_{k+1}}, n_{i_{k+2}}\right)$ means that either $n_{i_{k+1}} \neq n_{i_{k+2}}$ or $n_{i_{k+1}}=n_{i_{k+2}}$ but $\varepsilon_{k+1}=\varepsilon_{k+2}$. In the first case we show (assuming, as we may, $k=0$ ) that there are at most $3 \sqrt{N} \log N$ choices for 
the pair $\left(n_{i_{1}}, n_{i_{2}}\right)$ such that $n_{i_{1}} \neq n_{i_{2}}$ and the gap $n_{i_{2}} / n_{i_{3}}$ is large. Indeed, by the last assumption we have $n_{i_{3}} \leq n_{i_{2}} \cdot c /(32 p \sqrt{N}) \leq n_{i_{1}} \cdot c /(32 p \sqrt{N})$ and thus

$$
\left|\varepsilon_{3} n_{i_{3}}+\cdots+\varepsilon_{p} n_{i_{p}}\right| \leq n_{i_{1}} \cdot c /(32 \sqrt{N}) .
$$

On the other hand, by $(2.1)$ and $n_{i_{1}} \neq n_{i_{2}}$ we have $n_{i_{1}} / n_{i_{2}} \geq 1+c / \sqrt{i_{2}} \geq$ $1+c / \sqrt{N}$ and thus

$$
\begin{aligned}
2 n_{i_{1}} & \geq\left|\varepsilon_{1} n_{i_{1}}+\varepsilon_{2} n_{i_{2}}\right| \geq\left|n_{i_{1}}-n_{i_{2}}\right| \\
& \geq n_{i_{1}}\left(1-(1+c / \sqrt{N})^{-1}\right) \geq n_{i_{1}} \cdot c /(2 \sqrt{N}) .
\end{aligned}
$$

Hence the absolute value of the left side of $(2.5)$ lies in $\left[n_{i_{1}} \cdot c /(4 \sqrt{N}), 3 n_{i_{1}}\right]$ and thus (2.5) implies

$$
n_{i_{1}} \in[|d| / 3,4|d| \sqrt{N} / c] .
$$

Thus by Lemma 1 the number of choices for $n_{i_{1}}$ is at most

$$
\frac{2 \sqrt{N}}{c} \log \frac{12 \sqrt{N}}{c}+1 \leq 3 \sqrt{N} \log N \text {. }
$$

With $n_{i_{1}}$ chosen, there is at most one choice for $n_{i_{2}}$ by Lemma 3 since the gap $n_{i_{2}} / n_{i_{3}}$ is large. Thus in the first case of nontriviality of $\left(n_{i_{k+1}}, n_{i_{k+2}}\right)$ (i.e., $\left.n_{i_{k+1}} \neq n_{i_{k+2}}\right)$ the estimate in the third line of (2.8) is proved. In the second case, i.e., when $n_{i_{k+1}}=n_{i_{k+2}}$ but $\varepsilon_{k+1}=\varepsilon_{k+2}$, the proof is the same except that in this case (2.9) is replaced by $\left|\varepsilon_{1} n_{i_{1}}+\varepsilon_{2} n_{i_{2}}\right|=2 n_{i_{1}}$.

We turn now to the case $s \geq 3$ in (2.8). Again, we may assume $k=0$, i.e., we estimate the number of choices for the block $\left(n_{i_{1}}, \ldots, n_{i_{s}}\right)$. Fix integers $j_{2}, \ldots, j_{s-1}$ and first estimate the number of those blocks $\left(n_{i_{1}}, \ldots, n_{i_{s}}\right)$ where $n_{i_{\nu}} / n_{i_{\nu+1}} \asymp 2^{j_{\nu}}, 2 \leq \nu \leq s-1$. Clearly, for $n_{i_{1}}$ there are at most $N$ possibilities and given $n_{i_{1}}, \ldots, n_{i_{\nu-1}}, 2 \leq \nu \leq s-1$, Lemma 2 shows that for $n_{i_{\nu}}$ there are at most $(48 \sqrt{N} / c) \cdot \psi\left(j_{\nu}\right)$ possibilities where the function $\psi(j), j=0,1, \ldots$, is defined by

$$
\psi(j)= \begin{cases}1 & \text { if } p 2^{-j} \geq 1 / 8 \\ p 2^{-j} & \text { if } p 2^{-j}<1 / 8\end{cases}
$$

Finally, given $n_{i_{1}}, \ldots, n_{i_{s-1}}$, for $n_{i_{s}}$ there is at most one possibility by Lemma 3 since $\left(n_{i_{1}}, \ldots, n_{i_{s}}\right)$ is a block, i.e., the gap $n_{i_{s}} / n_{i_{s+1}}$ is large. (Again, if $s=p$ then Lemma 3 does not apply but for $n_{i_{p}}$ there is trivially at most one possibility if $n_{i_{1}}, \ldots, n_{i_{p-1}}$ are given.) Thus the number of choices for $\left(n_{i_{1}}, \ldots, n_{i_{s}}\right)$ is at most

$$
N(48 \sqrt{N} / c)^{s-2} \prod_{\nu=2}^{s-1} \psi\left(j_{\nu}\right) .
$$

Summing (2.10) for $j_{2}, \ldots, j_{s-1}$ we get an upper estimate for the number of blocks $\left(n_{i_{1}}, \ldots, n_{i_{s}}\right)$. Note that

$$
\sum_{j=0}^{\infty} \psi(j)=\sum_{p 2^{-j} \geq 1 / 8} 1+\sum_{p 2^{-j}<1 / 8} p 2^{-j} \leq 2 \log 8 p+1+1 / 4 \leq 12 \log p \quad(p \geq 2)
$$

since the last sum is a geometric series with ratio $1 / 2$ and first term $\leq 1 / 8$. Hence adding (2.10) for $j_{2}, \ldots, j_{s-1}$ we get at most

$$
N(48 \sqrt{N} / c)^{s-2} \prod_{\nu=2}^{s-1}\left(\sum_{j_{\nu=0}}^{\infty} \psi\left(j_{\nu}\right)\right) \leq N(48 \sqrt{N} / c)^{s-2}(12 \log p)^{s-2}
$$

proving the estimate in the first line of $(2.8)$. 
Lemma 5. The number of solutions of (2.5) containing a block of length 1 or a nontrivial block of length 2 is at most

$$
4(1200 \log p)^{p} N^{(p-1) / 2} \log N
$$

Proof. By Lemma 4 the number of solutions of (2.5) with block lengths $s_{1}, \ldots$, $s_{r}\left(s_{i} \geq 1, s_{1}+\cdots+s_{r}=p\right)$ is at most

$$
\begin{gathered}
\prod_{\left\{\nu: s_{\nu} \geq 3\right\}} \frac{(600 \log p)^{s_{\nu}}}{c^{s_{\nu}-2}} N^{s_{\nu} / 2} \cdot \prod_{\left\{\nu: s_{\nu}=1\right\}} 1 \cdot \prod_{\substack{\left\{\nu: \leq s_{\nu}=2 \text { and the } \\
\nu \text { th block is trivial }\right\}}} N \\
\cdot \prod_{\substack{\left\{\nu: s_{\nu}=2 \text { and the } \\
\nu \text { th block is nontrivial }\right\}}} 3 \sqrt{N} \log N .
\end{gathered}
$$

If there is a $\nu$ with $s_{\nu}=1$ then $\sum_{\left\{\nu: s_{\nu} \geq 2\right\}} s_{\nu} \leq p-1$ and thus using $c \geq 1$ and estimating $3 \sqrt{N} \log N$ by $N$ it follows that the expression in (2.12) is

$$
\leq \prod_{\left\{\nu: s_{\nu} \geq 3\right\}}(600 \log p)^{s_{\nu}} N^{s_{\nu} / 2} \cdot \prod_{\left\{\nu: s_{\nu}=2\right\}} N^{s_{\nu} / 2} \leq(600 \log p)^{p} N^{(p-1) / 2}
$$

On the other hand, if there is a nontrivial block of length 2 , i.e., the last product in (2.12) is not empty, then replacing all terms of the product by $N$ we increase the product by at least a factor $\sqrt{N} /(3 \log N)$ and thus the expression (2.12) is

$$
\leq \prod_{\left\{\nu: s_{\nu} \geq 3\right\}}(600 \log p)^{s_{\nu}} N^{s_{\nu} / 2} \prod_{\left\{\nu: s_{\nu}=2\right\}} N^{s_{\nu} / 2} \cdot \frac{3 \log N}{\sqrt{N}} \leq(600 \log p)^{p} N^{p / 2} \frac{3 \log N}{\sqrt{N}} .
$$

Since the number of systems $\left(s_{1}, \ldots, s_{r}\right)$ satisfying $1 \leq r \leq p, s_{i} \geq 1, s_{1}+$ $\cdots+s_{r}=p$ is at most $2^{p}$, the number of solutions of $(2.5)$ containing a block of length 1 or a nontrivial block of length 2 is bounded by the expression in (2.1), as stated.

In the sequel, call a solution $\left(n_{i_{1}}, \ldots, n_{i_{p}}\right)$ of $(2.5)$ regular if it contains only blocks of length $\geq 3$ and trivial blocks of length 2 .

Lemma 6. Let $L \geq 0$ and $p=s+t$ where $s \geq 0, t \geq 0$, and $s, t$ are even. Then the number of those regular solutions of (2.5) which contain $L$ blocks of length $\geq 3$ with total length $s$ and t/2 trivial blocks is at most

$$
\frac{1}{\left(\frac{t}{2}\right) ! L !} \frac{(1200 \log p)^{s}}{c^{s-2 L}} N^{p / 2}
$$

Proof. Fix integers $s_{1}, \ldots, s_{r} \geq 2$ such that $p=s_{1}+\cdots+s_{r}$. It is easy to see that the number of regular solutions of (2.5) with block lengths $s_{1}, \ldots, s_{r}$ is at most

$$
\frac{N^{r}}{r !} \prod_{\left\{\nu: s_{\nu} \geq 3\right\}} \frac{(600 \log p)^{s_{\nu}}}{c^{s_{\nu}-2}} N^{s_{\nu} / 2-1} .
$$

Indeed, fix the first element of each block. This means choosing $r$ different elements of the sequence $n_{1}, \ldots, n_{N}$, i.e., the number of choices is $\left(\begin{array}{l}N \\ r\end{array}\right) \leq$ $N^{r} / r$ !. Once these first elements are chosen, the trivial blocks are determined and by Lemma 4 the number of choices for the remaining elements in a block 
of length $s_{\nu} \geq 3$ (assuming that all elements preceding this block are already chosen) is at most

$$
\frac{(600 \log p)^{s_{\nu}}}{c^{s_{\nu}-2}} N^{s_{\nu} / 2-1} .
$$

(The extra -1 in the exponent of $N$ is due to the fact that in Lemma 4 the first element of the block was not fixed and we estimated the number of choices for this first element by $N$, while here the first element is fixed.) Hence the estimate (2.14) is verified.

Now let $s, t, L$ be given as in the formulation of the lemma and let $M_{s, t, L}$ be the number of regular solutions of (2.5) with parameters $s, t, L$ considered in Lemma 6. By the preceding estimates, we get an upper bound for $M_{s, t, L}$ if we add (2.14) for all systems $\left(s_{1}, \ldots, s_{r}\right)$ of integers $\geq 2$ satisfying $s_{1}+\cdots+$ $s_{r}=p$ and

$$
s=\sum_{\left\{\nu: s_{\nu} \geq 3\right\}} s_{\nu}, \quad t=p-s, \quad L=\sum_{\left\{\nu: s_{\nu} \geq 3\right\}} 1 .
$$

We observe first that the number of such systems $\left(s_{1}, \ldots, s_{r}\right)$ is at most

$$
\frac{\left(\frac{t}{2}+L\right) !}{\left(\frac{t}{2}\right) ! L !} 2^{s} \text {. }
$$

Indeed, such a system can be obtained in two steps. First we choose those indices $\nu, 1 \leq \nu \leq r$, for which $s_{\nu}=2$; since the number of such $\nu$ 's is $t / 2$ and $r=t / 2+L$, the number of choices is given by the fraction in (2.16). Once the indices $\nu$ with $s_{\nu}=2$ are given, we have to split $s$ in the form given by the first equation of (2.15). Clearly the number of possibilities is $\leq 2^{s}$, verifying the estimate (2.16).

Observe now that under the side condition (2.15) the estimate (2.14) becomes

$$
\frac{1}{r !} \frac{(600 \log p)^{s}}{c^{s-2 L}} N^{s / 2-L+r}=\frac{1}{\left(\frac{t}{2}+L\right) !} \frac{(600 \log p)^{s}}{c^{s-2 L}} N^{p / 2} .
$$

Multiplying this expression with (2.16) we get estimate (2.13), completing the proof of Lemma 6.

Note that in Lemmas 2-6 above we considered equation (2.5) where the signs $\varepsilon_{1}, \ldots, \varepsilon_{p}$ were fixed and the indices $i_{1}, \ldots, i_{p}$ were in nonincreasing order. Permuting the indices $i_{1}, \ldots, i_{p}$ and choosing the signs $\varepsilon_{1}, \ldots, \varepsilon_{p}$ in all possible ways we get an estimate for the number of solutions of the original equation (2.3):

Lemma 7. Let $L \geq 0$ and $p=s+t$ where $s \geq 0, t \geq 0$, and $s, t$ are even. Then the number of those regular solutions of (2.3) which contain $L$ blocks of length $\geq 3$ with total length $s$ and t 2 trivial blocks is at most

$$
\frac{p !}{\left(\frac{t}{2}\right) ! L !} \frac{(2400 \log p)^{s}}{c^{s-2 L}} N^{p / 2} \text {. }
$$

On the other hand, the number of nonregular solutions of (2.3) is at most

$$
4 p !(2400 \log p)^{p} N^{(p-1) / 2} \log N .
$$

Here, a solution of (2.3) is called regular if it becomes regular after arranging $i_{1}, \ldots, i_{p}$ in a nonincreasing fashion. Also, the blocks in a solution of $(2.3)$ are meant after this rearrangement. 
Proof of Lemma 7. Since the number of permutations of $i_{1}, \ldots, i_{p}$ is $\leq$ $p !\left(i_{1}, \ldots, i_{p}\right.$ are not necessarily different $)$ and the number of choices for $\varepsilon_{1}, \ldots, \varepsilon_{p}$ is $2^{p}$, estimate $(2.18)$ is immediate from Lemma 5 . To deduce (2.17) from Lemma 6 consider the regular solutions of (2.5) with block lengths $s_{1}, \ldots, s_{r}$. In a block of length $s_{\nu} \geq 3$ there are $2^{s_{\nu}}$ different choices for the signs $\varepsilon_{i}$ while in a trivial block of length 2 there are only 2 choices (instead of 4 ) since the two signs in a trivial block are opposite. Hence if $t / 2$ is the number of trivial blocks and $s=p-t$ then the total number of choices for the signs $\varepsilon_{1}, \ldots, \varepsilon_{p}$ is $\leq 2^{s} 2^{t / 2}$. On the other hand, among the indices $i_{1}, \ldots, i_{p}$ there are $t / 2$ pairs of equal numbers and thus the number of different permutations of $i_{1}, \ldots, i_{p}$ is $\leq p ! / 2^{t / 2}$. Thus passing from equation (2.5) to (2.3) means an extra factor $\leq p ! 2^{s}$ in (2.13), proving (2.17).

Using Lemma 7 it is now easy to complete the proof of the Main Lemma. Let us note first that in Lemma $7 s$ is the total length of $L$ blocks of length $\geq 3$ and thus $s \geq 3 L$; on the other hand, for $(t / 2)$ ! appearing in (2.17) we have

$$
\left(\frac{t}{2}\right) !=\left(\frac{p}{2}-\frac{s}{2}\right) ! \geq \frac{\left(\frac{p}{2}\right) !}{p^{s / 2}} .
$$

Hence using $c \geq p^{1 / 2+\varepsilon}$ we get that the expression in (2.17) cannot exceed

$$
\begin{aligned}
\frac{p ! p^{s / 2}}{\left(\frac{p}{2}\right) ! L !} & \frac{(2400 \log p)^{s}}{p^{s / 2-L} p^{(s-2 L) \varepsilon}} N^{p / 2} \leq \frac{p^{L}}{L !} \frac{(2400 \log p)^{s}}{p^{s \varepsilon / 3}} \frac{p !}{(p / 2) !} N^{p / 2} \\
\quad \leq & \frac{p^{s(1-\varepsilon) / 3}}{[s / 3] !}(2400 \log p)^{s} \frac{p !}{(p / 2) !} N^{p / 2}=\frac{\tau^{s}}{[s / 3] !} \frac{p !}{(p / 2) !} N^{p / 2}
\end{aligned}
$$

where [ ] denotes integral part and

$$
\tau=2400 p^{(1-\varepsilon) / 3} \log p .
$$

(In the second inequality of (2.19) we used the fact that $p^{n} / n$ ! is increasing for $0 \leq n \leq p-1$.) Now it is easy to see that

$$
\sum_{s=0}^{\infty} \frac{(s+1) \tau^{s}}{[s / 3] !} \leq \exp \left(2 \tau^{3}\right), \quad \tau \geq \tau_{0},
$$

and thus adding the last expression in (2.19) for $0 \leq L \leq s / 3$ and then for $s=0,1,2, \ldots$ we get that the number of regular solutions of $(2.3)$ is

$$
\leq \exp \left\{K p^{1-\varepsilon} \log ^{3} p\right\} \frac{p !}{(p / 2) !} N^{p / 2}
$$

provided that $p \geq K$ where $K$ is an absolute constant. On the other hand, by the second statement of Lemma 7 the number of nonregular solutions of (2.3) is

$$
\begin{aligned}
& \leq 4 \frac{p !}{(p / 2) !}(2400 p \log p)^{p} N^{p / 2-1 / 4} \leq \frac{p !}{(p / 2) !} e^{p^{2}} N^{p / 2-1 / 4} \\
& \leq e^{-p^{2}} \frac{p !}{(p / 2) !} N^{p / 2}
\end{aligned}
$$

for $N \geq K, K \leq p \leq 4 \log \log N$ since $e^{2 p^{2}} \leq N^{1 / 4}$. This completes the proof of the Main Lemma for $\delta=1$. 
To get the Main Lemma for $0<\delta<1$ let us observe that if $i_{1}, \ldots, i_{p}$ are restricted to the interval $[(1-\delta) N, N]$ instead of $[0, N]$ then the estimates in the first and last lines of $(2.8)$ have to be multiplied with $\delta$ (in fact, in the general case we have $\delta N$ possibilities for the first element of each block instead of $N$ ). As a consequence, estimates (2.13) and (2.17) have to be multiplied with $\delta^{L+t / 2} \leq \delta^{t / 2}=\delta^{p / 2-s / 2}$. Hence estimate (2.19) will hold in the general case if $N^{p / 2}$ is replaced by $(\delta N)^{p / 2}$ and $(2400 \log p)^{s}$ is replaced by $(2400 \log p / \sqrt{\delta})^{s}$, leading to a new value

$$
\tau=2400 p^{1-\varepsilon} \log ^{3} p / \sqrt{\delta}
$$

giving the estimate (2.4). On the other hand, estimate (2.18) for the number of nonregular solutions of $(2.3)$ remains valid for general $\delta$ and from the second condition of (2.2) (written equivalently as $N \geq \exp (\exp (p / 4)))$ it follows that for $N \geq N_{0}(\delta)$ we have

$$
4 p !(2400 \log p)^{p} \leq \delta^{p / 2} N^{1 / 4} .
$$

Hence the expression in (2.18) is also bounded by (2.4), completing the proof of the Main Lemma for general $\delta$.

In the above proof we assumed $0<\varepsilon \leq 1$, i.e., the number $\tau$ in (2.20) is large for $p$ large. For $\varepsilon>1$ the situation changes essentially: in this case $\tau=O\left(p^{-(\varepsilon-1) / 6}\right)$ is small for $p$ large and the sum of the power series (2.21) becomes $1+O(\tau)=1+O\left(p^{-(\varepsilon-1) / 6}\right)$. Moreover, if we exclude the trivial solutions of (2.3) (this makes a difference only for $d=0$ ) then in Lemmas 6 and $7 s$ cannot be 0 and thus the sum in $(2.21)$ becomes $O(\tau)=O\left(p^{-(\varepsilon-1) / 6}\right)$. Since the number of nonregular solutions of $(2.3)$ is bounded by the expression in (2.22), it follows that for $\varepsilon>1$ the factor $\exp \left\{K p^{1-\varepsilon} \log ^{3} p / \sqrt{\delta}\right\}$ in (2.4) can be replaced by $1+O\left(p^{-(\varepsilon-1) / 6}\right)$ or $O\left(p^{-(\varepsilon-1) / 6}\right)$ according as we include or exclude the trivial solutions. In other words, for $\varepsilon>1$ and $p$ large, almost all solutions of (2.3) with $d=0$ are trivial.

In conclusion we note that although the gap condition (2.1) in the Main Lemma is assumed for all $1 \leq j \leq N-1$, our proof used it only for $(1-\delta) N \leq$ $j \leq N-1$ (i.e., for the values actually appearing in (2.3)). Similarly, if we assume (2.1) for $(1-\delta) N+a \leq j \leq N-1$ then estimate (2.4) will be valid for the number of solutions of

$$
\pm n_{i_{1}} \pm \cdots \pm n_{i_{p}}=d, \quad(1-\delta) N+a \leq i_{1}, \ldots, i_{p} \leq N .
$$

\section{Proof of THE THEOREM}

Using the Main Lemma of $\S 2$, the proof our our theorem can be completed in a rather standard way. We first note the following consequence of the Main Lemma:

Lemma 8. Let $\left\{n_{j}, 1 \leq j \leq N\right\}$ be a finite sequence of positive integers satisfying

$$
n_{j+1} / n_{j} \geq 1+c / \sqrt{j}, \quad(1-\delta) N \leq j \leq N-1 .
$$

Further let $p \geq 2$ be an even integer and assume that (2.2) holds for some $\varepsilon>0$.

Then for $N \geq N_{0}(\delta), p \geq K$ we have

$$
\int_{0}^{2 \pi}\left(\sum_{(1-\delta) N \leq j \leq N} \cos n_{j} x\right)^{p} d x \leq 2 \pi 2^{-p} A_{N, p, \delta}
$$


where $A_{N, p, \delta}$ is the number in (2.4). The result remains valid if the lower limit $(1-\delta) N$ in (3.1) and in the sum in (3.2) is replaced by $(1-\delta) N+a$, provided this number is $\leq N$.

To see this, let us note that the integrand in (3.2) equals

$$
2^{-p} \sum \cos \left( \pm n_{i_{1}} \pm \cdots \pm n_{i_{p}}\right) x
$$

where the summation is extended for all $p$-tuples $\left(i_{1}, \ldots, i_{p}\right)$ with $(1-\delta) N \leq$ $i_{1}, \ldots, i_{p} \leq N$ and all choices of the signs \pm 1 . Since for integer $n$ we have $\int_{0}^{2 \pi} \cos n x d x=2 \pi$ or 0 according as $n=0$ or $n \neq 0$, the result follows from the Main Lemma. (See the remark at the end of $\S 2$.)

We shall also need the following maximal inequality for trigonometric sums, proved in our paper [1]:

Lemma 9. Let $p \geq 2$ be an even integer and $f \in L^{p}(0,2 \pi)$ an even function with nonnegative Fourier coefficients. Let $s_{n}(f)$ denote the nth partial sum of the Fourier series of $f$. Then

$$
\int_{0}^{2 \pi}\left(\sup _{k \geq 1}\left|s_{2^{k}}(f)\right|\right)^{p} d x \leq A^{p} \int_{0}^{2 \pi}|f|^{p} d x
$$

where $A$ is an absolute constant.

Now let $\left(n_{k}\right)$ be a sequence of positive integers satisfying (1.5) with $\alpha=$ $1 / 2+\varepsilon, \varepsilon>0$. Let $N \geq 1, \lambda>1$ and set

$$
p=2[\log \log N], \quad c=(\log \log \sqrt{N})^{1 / 2+\varepsilon} .
$$

Then we have

$$
n_{j+1} / n_{j} \geq 1+c / \sqrt{j}, \quad \sqrt{N} \leq j \leq N-1 ;
$$

further (2.2) holds for $N \geq N_{0}$ with $\varepsilon$ replaced by $\varepsilon / 2$. Hence applying Lemma 8 with $\delta=1, a=\sqrt{N}$ and using $p ! /(p / 2) ! \sim \sqrt{2}(2 p / e)^{p / 2}$ and the Markov inequality, we get

$$
\begin{aligned}
\lambda\{0 & \left.\leq x \leq 2 \pi:\left|\sum_{\sqrt{N} \leq k \leq N} \cos n_{k} x\right|>(\lambda N \log \log N)^{1 / 2}\right\} \\
& \leq(\lambda N \log \log N)^{-p / 2} 2 \pi 2^{-p} \exp \left\{K p^{1-\varepsilon / 4}\right\} \frac{p !}{(p / 2) !} N^{p / 2} \\
& \leq \lambda^{-p / 2}(p / 2)^{-p / 2} 2 \pi 2^{-p} \exp \left\{K p^{1-\varepsilon / 4}\right\} 2(2 p / e)^{p / 2} \\
& =4 \pi(\lambda e)^{-p / 2} \exp \left\{K p^{1-\varepsilon / 4}\right\} \\
& \leq 4 \pi \exp \left\{-(1+\log \lambda)[\log \log N]+2 K[\log \log N]^{1-\varepsilon / 4}\right\} \\
& \leq \exp \{-(1+\tau) \log \log N\}
\end{aligned}
$$

for $N \geq N_{0}$ where $\tau=\frac{1}{2} \log \lambda>0$. Thus setting $S_{N}=\sum_{k \leq N} \cos n_{k} x$ and choosing $N_{k}=\left[a^{k}\right], 1<a<2$, it follows from the above estimates and the Borel-Cantelli lemma that

$$
\limsup _{k \rightarrow \infty} \frac{\left|S_{N_{k}}-S_{\left[N_{k}^{1 / 2}\right]}\right|}{\left(N_{k} \log \log N_{k}\right)^{1 / 2}} \leq \sqrt{\lambda} \quad \text { a.e. }
$$


Clearly $\left|S_{\left[N_{k}^{1 / 2}\right]}\right| \leq N_{k}^{1 / 2}$ and thus the last relation implies

$$
\limsup _{k \rightarrow \infty} \frac{\left|S_{N_{k}}\right|}{\left(N_{k} \log \log N_{k}\right)^{1 / 2}} \leq \sqrt{\lambda} \quad \text { a.e. }
$$

Since $\lambda$ and $a$ can be chosen arbitrarily close to 1 , our theorem will follow if we show that setting

$$
M_{k}=\max _{N_{k} \leq j \leq N_{k+1}}\left|S_{j}-S_{N_{k}}\right|
$$

we have

$$
\limsup _{k \rightarrow \infty} \frac{M_{k}}{\left(N_{k} \log \log N_{k}\right)^{1 / 2}} \leq 8 A(a-1)^{1 / 2} \quad \text { a.e. }
$$

where $A$ is the absolute constant appearing in Lemma 9.

To prove (3.3) set

$$
Z_{k}=\sum_{j=N_{k}+1}^{N_{k+1}} \cos n_{j} x, \quad p(k)=\max \left\{i: n_{i} \leq 2^{k}\right\}, \quad H=\{p(1), p(2), \ldots\} .
$$

Clearly

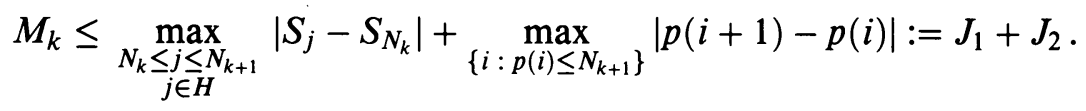

Using (1.5) with $\alpha>1 / 2$ we get for $k \geq k_{0}$

$$
\begin{aligned}
2 & \geq n_{p(k+1)} / n_{p(k)+1} \geq \prod_{m=p(k)+1}^{p(k+1)-1}(1+1 / \sqrt{m}) \\
& \geq 1+\sum_{m=p(k)+1}^{p(k+1)-1} 1 / \sqrt{m} \geq 1+(p(k+1)-p(k)-1) / \sqrt{p(k+1)}
\end{aligned}
$$

whence it follows that $p(k+1) / p(k) \rightarrow 1$ and

$$
p(k+1)-p(k)=O\left(p(k)^{1 / 2}\right) .
$$

Thus for $J_{2}$ in (3.4) we have

$$
\left|J_{2}\right|=O\left(N_{k+1}^{1 / 2}\right)=o\left(N_{k} \log \log N_{k}\right)^{1 / 2} .
$$

On the other hand, choosing $p=2\left[\log \log N_{k}\right]$ and applying Lemma 9 with $f=Z_{k}$ we get

$$
\int_{0}^{2 \pi}\left|J_{1}\right|^{p} d x \leq A^{p} \int_{0}^{2 \pi}\left|Z_{k}\right|^{p} d x
$$

where $A$ is an absolute constant. Using (1.5) with $\alpha>1 / 2$ it follows that relation (3.1) holds with $N=N_{k+1}, c=\left(\log \log N_{k}\right)^{1 / 2+\varepsilon}, \delta=1-1 / a$ and (2.2) is also valid for $k \geq k_{0}$, with $\varepsilon$ replaced by $\varepsilon / 2$. Hence by Lemma 8 the integral on the right-hand side of (3.6) is at most

$$
2 \pi 2^{-p} \exp \left\{\frac{K p^{1-\varepsilon / 4}}{\sqrt{1-1 / a}}\right\} \frac{p !}{(p / 2) !} 2\left(N_{k+1}-N_{k}\right)^{p / 2} .
$$


Hence (3.6) and the Markov inequality imply for $k \geq k_{0}$

$$
\begin{aligned}
\lambda\{0 \leq & \left.x \leq 2 \pi:\left|J_{1}\right| \geq 4 A\left(N_{k+1}-N_{k}\right)^{1 / 2}\left(\log \log N_{k}\right)^{1 / 2}\right\} \\
\leq & (4 A)^{-p}\left(N_{k+1}-N_{k}\right)^{-p / 2}\left(\log \log N_{k}\right)^{-p / 2} A^{p} 2 \pi 2^{-p} \\
& \cdot \exp \left\{\frac{K p^{1-\varepsilon / 4}}{\sqrt{1-1 / a}}\right\} \frac{p !}{(p / 2) !} 2\left(N_{k+1}-N_{k}\right)^{p / 2} \\
\leq & 4^{-p}(p / 2)^{-p / 2} 2 \pi 2^{-p} \exp (p / 2) 4(2 p / e)^{p / 2} \leq 8 \pi 4^{-p} \leq k^{-3 / 2} .
\end{aligned}
$$

Hence by the Borel-Cantelli lemma and $N_{k+1}-N_{k} \sim(a-1) N_{k}$ we have

$$
\left|J_{1}\right| \leq 8 A(a-1)^{1 / 2}\left(N_{k} \log \log N_{k}\right)^{1 / 2} \quad \text { a.e. for } k \geq k_{0} .
$$

Now (3.4), (3.5), and (3.7) imply (3.3), completing the proof of the theorem.

\section{Remarks}

In this section we construct an example showing that for small enough $\varepsilon>0$ the subexponential factor

$$
\exp \left\{\frac{K p^{1-\varepsilon} \log ^{3} p}{\sqrt{\delta}}\right\}
$$

in (2.4) cannot be replaced by $O(1)$ or even by $o\left(p^{2-2 \varepsilon}\right)$. Let $a_{k}=(k !)^{2}$, $m_{k}=\left[k /(\log \log k)^{2 \alpha}\right] \quad(k \geq 3), m_{1}=m_{2}=0$, and $M_{k}=\sum_{i=1}^{k} m_{i}$ for some $\alpha>1 / 2$. Let $I_{k}=\left\{a_{k}, 2 a_{k}, \ldots, m_{k} a_{k}\right\}$; clearly the sets $I_{k}, k=1,2, \ldots$, are disjoint. Define the sequence $\left(n_{k}\right)$ by $\left(n_{k}\right)=\bigcup_{j=1}^{\infty} I_{j}$. It is easily seen that

$$
\frac{n_{j+1}}{n_{j}} \geq 1+\frac{(\log \log j)^{\alpha}}{2 \sqrt{j}}, \quad j \geq j_{0} .
$$

Indeed, if $M_{k-1}<j<M_{k}$ then setting $i=j-M_{k-1}$ and using

$$
M_{k} \sim \frac{k^{2}}{2(\log \log k)^{2 \alpha}} \quad(k \rightarrow \infty)
$$

and $M_{k} / M_{k-1} \rightarrow 1$ we get

$$
\begin{aligned}
\frac{n_{j+1}}{n_{j}} & =1+\frac{1}{i} \geq 1+\frac{1}{m_{k}} \geq 1+\frac{(\log \log k)^{2 \alpha}}{k} \geq 1+\frac{\left(\log \log M_{k}\right)^{\alpha}}{2 \sqrt{M_{k-1}}} \\
& \geq 1+\frac{(\log \log j)^{\alpha}}{2 \sqrt{j}}, \quad j \geq j_{0} .
\end{aligned}
$$

On the other hand, if $j=M_{k}$ then $n_{j+1} / n_{j}=a_{k+1} /\left(m_{k} a_{k}\right) \geq 2$; i.e., (4.1) holds in this case too. Let $N=M_{k}, 0<\delta \leq 1$; we show that the number of nontrivial solutions of

$$
\pm n_{i_{1}} \pm \cdots \pm n_{i_{p}}=0, \quad(1-\delta) N \leq i_{1}, \ldots, i_{p} \leq N,
$$

is at least

$$
\text { const } \cdot p^{2-2 \alpha} \frac{p !}{(p / 2) !}(\delta N)^{p / 2}
$$

for any even number $p$ satisfying $\log \log N \leq p \leq 4 \log \log N$. We consider again the case $\delta=1$; for general $\delta$ the proof is similar. Let us call a solution 
$\left(n_{i_{1}}, \ldots, n_{i_{p}}\right), 1 \leq i_{1} \leq i_{2} \leq \cdots \leq i_{p} \leq N$ of (4.2) (where we take $\delta=1$ ) almost trivial if the $n_{i_{\nu}}$ 's in (4.2) are pairwise equal with opposite signs except one 4-tuple $n_{i_{s_{1}}}<n_{i_{s_{2}}}<n_{i_{s_{3}}}<n_{i_{s_{4}}}$ belonging to the set $I_{l}$ for some $1 \leq l \leq k$ and satisfying

$$
n_{i_{s_{1}}}+n_{i_{s_{2}}}+n_{i_{s_{3}}}-n_{i_{s_{4}}}=0 .
$$

Moreover, we stipulate that the elements of different pairs are different and they all differ from the elements of the 4-tuple $\left(n_{i_{s_{1}}}, n_{i_{s_{2}}}, n_{i_{s_{3}}}, n_{i_{s_{4}}}\right)$. Since $I_{l}=\left\{j a_{l}, 1 \leq j \leq m_{l}\right\}$, clearly for each triplet $\left(n_{i_{s_{1}}}, n_{i_{s_{2}}}, n_{i_{s_{3}}}\right)$ lying in $I_{l}$ and satisfying $n_{i_{s_{1}}}<n_{i_{s_{2}}}<n_{i_{s_{3}}}<a_{l} m_{l} / 3$ there exists a unique $n_{i_{s_{4}}}$ in $I_{l}$ such that (4.4) holds. Thus the number of 4-tuples $n_{i_{s_{1}}}<n_{i_{s_{2}}}<n_{i_{s_{3}}}<n_{i_{4}}$ lying in $I_{l}$ and satisfying (4.4) is at least const $\cdot m_{l}^{3}$. Hence in an almost trivial solution the number of choices for the exceptional 4-tuple $\left(n_{i_{s_{1}}}, n_{i_{s_{2}}}, n_{i_{s_{3}}}, n_{i_{s_{4}}}\right)$ is at least

$$
\text { const } \cdot \sum_{l=1}^{k} m_{l}^{3} \sim \text { const } \cdot \frac{k^{4}}{(\log \log k)^{6 \alpha}} \sim \text { const } \cdot \frac{N^{2}}{(\log \log N)^{2 \alpha}} \geq \text { const } \cdot \frac{N^{2}}{p^{2 \alpha}} \text {. }
$$

Once $\left(n_{i_{1}}, n_{i_{s_{2}}}, n_{i_{s_{3}}}, n_{i_{s_{4}}}\right)$ is fixed, the number of choices for the remaining $(p-4) / 2$ pairs clearly equals

$$
\left(\begin{array}{c}
N-4 \\
(p-4) / 2
\end{array}\right) \geq \frac{(N-p)^{p / 2-2}}{(p / 2-2) !} \geq \frac{p^{2}}{8} \frac{N^{p / 2-2}}{(p / 2) !}
$$

for $N \geq N_{0}$ since $p \leq 4 \log \log N$ implies $(N-p)^{p / 2-2} \sim N^{p / 2-2}$. Thus the total number of choices for the almost trivial solution $\left(n_{i_{1}}, \ldots, n_{i_{p}}\right), 1 \leq i_{1} \leq$ $i_{2} \leq \cdots \leq i_{p} \leq N$ is

$$
\geq \text { const } \cdot p^{2-2 \alpha} \frac{N^{p / 2}}{(p / 2) !} .
$$

Since the number of choices for the signs in (4.2) is at least $2^{p / 2-2}$ (in each pair $\left(n_{i_{q}}, n_{i_{r}}\right)$ with $n_{i_{q}}=n_{i_{r}}$ there are two choices of the signs $\varepsilon_{i_{q}}, \varepsilon_{i_{r}}$ such that $\varepsilon_{i_{q}}=-\varepsilon_{i_{r}}$ and since the number of permutations of $\left(n_{i_{1}}, \ldots, n_{i_{p}}\right)$ is $p ! / 2^{p / 2-2}$, our estimate (4.3) is proved.

In view of the proof of Lemma 8, estimate (4.3) with $\delta=1$ implies that

$$
\int_{0}^{2 \pi}\left(\sum_{k \leq N} \cos n_{k} x\right)^{p} d x \geq \text { const } \cdot p^{2-2 \alpha} \frac{p !}{2^{p}(p / 2) !} N^{p / 2}
$$

for $\log \log N \leq p \leq 4 \log \log N$. On the other hand, observe that if $\xi_{k}, k=$ $1,2, \ldots$, are independent normal random variables having the same mean and variance as $\cos n_{k} x$ then

$$
E\left(\sum_{k \leq N} \xi_{k}\right)^{p}=\frac{p !}{2^{p}(p / 2) !} N^{p / 2}, \quad p=2,4, \ldots
$$

Thus (4.5) expresses the surprising fact that under (1.5), $\alpha>1 / 2$ the asymptotic behavior of the high moments of $\sum_{k \leq N} \cos n_{k} x$ can be different from that of sums of i.i.d. r.v.'s even though $\left(\cos n_{k} x\right)$ satisfies the central limit theorem (1.2) and the law of the iterated logarithm (1.6). This is another example of 
the unusual properties of $\left(\cos n_{k} x\right)$ under $(1.5)$, discussed in $\S 1$. On the other hand, in $\S 2$ we proved that for $\varepsilon>1$ the number of nontrivial solutions of (2.3) is $O\left(p^{-(\varepsilon-1) / 6}\right)$ and thus under (1.5) with $\alpha>3 / 2$ we have

$$
\frac{1}{2 \pi} \int_{0}^{2 \pi}\left(\sum_{k \leq N} \cos n_{k} x\right)^{p} \sim \frac{p !}{2^{p}(p / 2) !} N^{p / 2}
$$

as $N \rightarrow \infty$, uniformly for $2 \leq p \leq 4 \log \log N$. Thus for $\alpha>3 / 2$ the above pathological moment behavior cannot occur.

\section{REFERENCES}

1. I. Berkes, Critical LIL behavior of the trigonometric system, Trans. Amer. Math. Soc. 338 (1993), 553-585.

2. P. Erdős, On trigonometric sums with gaps, Magyar Tud. Akad. Mat. Kut. Int. Közl. 7 (1962), 37-42.

3. P. Erdös and I. S. Gál, On the law of the iterated logarithm, Nederl. Akad. Wetensch. Proc. Ser. A 58 (1955), 65-84.

4. W. Feller, The general form of the so-called law of the iterated logarithm, Trans. Amer. Math. Soc. 54 (1943), 373-401.

5. R. Salem and A. Zygmund, On lacunary trigonometric series, Proc. Nat. Acad. Sci. U.S.A. 33 (1947), 333-338.

6. _ـ La loi du logarithme itéré pour les séries trigonométriques lacunaires, Bull. Sci. Math. (2) 74 (1950), 209-224.

7. S. Takahashi, On the law of the iterated logarithm for lacunary trigonometric series, Tôhoku Math. J. 24 (1972), 319-329.

8. On the law of the iterated logarithm for lacunary trigonometric series. II, Tôhoku Math. J. 27 (1975), 391-403.

Mathematical Institute of the Hungarian Academy of Sciences, H-1364 Budapest, ReÁltanoda U. 13-15, Hungary

E-mail address: h1127ber@ella.hu 